\title{
STRIKING DIFFERENCES IN PERFORMANCE PATTERNS AND \\ NEUROPSYCHOLOGICAL CORRELATES WITHIN SEMANTIC AND PHONEMIC \\ FLUENCY TASKS
}

Petar Gabrić*, ORCiD 0000-0003-2644-194X, Institute for German Linguistics, Philipps University of Marburg, Marburg an der Lahn, Germany, petar.gabric@uni-marburg.de, petar.gabric1@gmail.com

Mija Vandek, ORCiD 0000-0003-3398-7350, Zagreb, Croatia, mijavandek@gmail.com

*corresponding author 
Abstract: Despite both theoretical and empirical linguistics suggesting otherwise, researchers using semantic and phonemic fluency tasks have uncritically assumed that there are no categoryor phoneme/letter-specific effects on verbal fluency performance. We recruited 16 young adult subjects and administered two semantic (animals, trees) and two phonemic $(K, M)$ fluency tasks. Executive functions were assessed using the Wisconsin Card Sorting Test (WCST). On the animal compared to the tree task, subjects produced significantly more legal words, had a significantly lower intrusion rate, significantly shorter first-response latencies and final silence periods, as well as significantly shorter between-cluster response latencies. These differences can at least partly be explained by differences in the category sizes, integrity of the categories' borders, and efficiency of the functional connectivity between subcategories. Switching on the animal but clustering on the tree task were moderately and strongly, respectively, correlated with the WCST. On the $K$ compared to the $M$ task, subjects produced significantly more legal words and had significantly shorter between-cluster response times. Counterintuitively, a corpus analysis revealed there are more words starting with the $(m)$ compared to $(k)$ in the experimental language. Performances on the $K$ and $M$ tasks were very limitedly associated with the WCST. Our results have important implications for research utilizing verbal fluency, indicating that researchers should pay close attention to the types of semantic categories and phonemes/letters within neuropsychological assessments, as well as in the context of reviews and meta-analyses. In order to accomplish this adequately, further research on the specificities of different verbal fluency tasks is direly needed.

Keywords: semantic fluency; phonemic fluency; clustering; neuropsychological assessment; behavioral methods; hypernymy 
Word count: 4939 (inclusive of abstract, tables, figures, and references) 


\section{INTRODUCTION}

Verbal fluency (VF) is a common neuropsychological task used for assessing linguistic and cognitive abilities in a range of human populations. Most commonly, the semantic (SF) and phonemic fluency (PF) tasks are administered, in which the subject is to name as many words of the given semantic category (e.g., fruits) or beginning with the given letter/phoneme (e.g., $F$ ), respectively. Research on SF and PF has demonstrated that the two task types reflect different lexical processes. Subjects tend to cluster successive responses according to their membership to a particular semantic subcategory of the given category on SF (e.g., subcategory wild cats of the category animals), i.e., according to the equivalence of the incipient or ending consonant-vowel structures on PF (e.g., words starting with $m a$ - on the letter $M$ fluency) (Troyer et al. 1997). The former reflects the structure and processes of semantic memory which is organized in semantic categories whose concepts are in either hypernymic/hyponymic or co-hyponymic hierarchical relationship with one another. The latter reflects the structure of the putative orthographic/phonological lexicon which should then be organized according to similarities in the consonant-vowel structures representing the form of the words.

\subsection{Category-specific and letter-specific effects on verbal fluency performance}

Most researchers utilizing SF and PF uncritically assume that there are no category-specific or phoneme/letter-specific effects on the quantity and quality of performance on different SF and PF tasks, as evidenced in both experimental and meta-analytical studies (e.g., Raucher-Chéné et al. 2017; Tan et al. 2020; Thiele et al. 2016). However, neuroscientific research has clearly 
demonstrated that semantic memory is widely distributed over the cortical surface and different semantic categories may be associated with disproportionate levels of metabolic activity in different brain regions (Huth et al. 2016). Furthermore, both theoretical and experimental linguistics agree that different lexical-semantic features such as concreteness/abstractness and animacy/inanimacy underlie divergent cognitive processing mechanisms (Ventura et al. 2005), while pathological populations may exhibit feature-specific deficits on lexical tasks (Carammaza \& Shelton 1998; Sekulić Sović et al. 2019). Similarly, processing of different phonological categories is associated with different cognitive and neural mechanisms (Lago et al. 2015).

Despite this, the effect of the given category or phoneme/letter on performance on SF and PF has been alarmingly understudied. It is clear from studies reporting descriptive data of multiple SF tasks that there are stark discrepancies in the number of legal words produced by healthy subjects across different semantic categories. Typically, adult healthy subjects produce the most words on animal fluency, yet productivity on other semantic categories may be reduced more than twofold. For example, Jebahi et al. (2020) reported that subjects produced 18 words on average on animal fluency but less than ten on the categories accessories, tools, transportation means, furniture, and sports. Moreover, pathological populations may show deficits of different degrees on different SF categories or they may show normative deficits on one but not the other category. Several studies on SF in dementia and Alzheimer's disease have revealed that the extent of the deficit is dependent on the given category's size (Diaz et al. 2004), while Moreno-Martínez et al. (2017) and Neves et al. (2020) found that although the animal fluency is by far the most commonly utilized SF task for research on dementia, other categories such as supermarket items, fruits, and vegetables are more sensitive in distinguishing healthy people from patients with dementia. On the other hand, Gabrić and Vandek (2020, in review) reported that patients with first-episode psychosis displayed 
deficient clustering on the categories animals, vegetables, and musical instruments but not on trees and fruits (cf. Vandek et al. 2018), while Tagini et al. (2018) reported that patients with Parkinson's disease showed semantically disorganized output on the category fruits but not animals. Similarly, there is a long-standing division between "easy" and "difficult" letters in English (Borkowski et al. 1967), recently confirmed by Barry et al. (2008) who found in a meta-analysis that performance on the CFL version of the Controlled Oral Word Association Test (COWAT) was significantly reduced and thus more difficult compared to the FAS version, as well as that healthy subjects exhibit greater variability on the FAS compared to the CFL version.

\subsection{Executive functioning and verbal fluency}

Although it is often assumed that VF tasks can be used as a measurement for testing executive functions (EFs), it is still unclear which subdomains of EFs have the greatest impact on VF tasks. Neuroimaging and focal lesion studies have shown that VF performance is closely related to frontal-lobe structures that are associated with EFs (Whiteside et al. 2016). To successfully solve the VF task, one must follow the category or initial letter rule, retrieve words from long-term memory, and avoid repetitions.

Studies report ambiguous results regarding the relationship between core EFs (i.e., working memory, inhibitory control, and cognitive flexibility) and VF performance (Amunts et al. 2020). While some studies connect VF performance to specific EFs, such as cognitive flexibility (Diamond 2013) that is usually measured with various task-switching and set-shifting tasks (e.g.,

Wisconsin Card Sorting Test), others question the link between EFs and VF performance (Henry and Crawford 2005). Whiteside et al. (2016) suggest that the critical component of VF tasks 
(animal and FAS fluency tasks) is not related to performance on EFs (i.e., cognitive flexibility tested with WCST and TMT-B), but language processing. Furthermore, Henry et al. (2015) found that language ability is associated with both PF (FAS) and SF (animals, boys' names). On the other hand, the link between VF tasks and EFs (i.e., executive-loaded working memory, inhibition, and switching) was modest as the only measure to show relations with VF was inhibition. However, it is important to emphasize the broadness of EFs, and the necessity of employing different neuropsychological measures to collect valid results. Therefore, Aita et al. (2018) incorporated various measures of EFs including fluid reasoning, working memory, shifting, and updating, while using the same methodology for both PF and SF tasks as Whiteside et al. The findings of these two studies were not consistent, since Aita et al. showed that multiple aspects of EFs were significantly related to both PF and SF. Regarding cognitive flexibility, Amunts et al. (2020) pointed to significant relation between multiple tests of cognitive flexibility (i.e., WCST and TMT) and VF performance (animals, jobs). Importantly, this study used a time span of 2 minutes, while other studies mentioned here used 60 seconds, which is more common.

While Luo et al. (2010) indicated that executive control is more important for the PF task (FAS) than the SF task (clothing items, girls' names), Shao et al. (2014) were not able to replicate these results (PF: M, S; SF: animals, food). Moreover, Aita et al. (2018) have found stronger relations between SF and the EFs factors compared to PF. Consequently, further research is needed to determine the nature of the link between executive control and specific fluency tasks.

\section{METHODS}




\subsection{Subjects}

Sixteen subjects were recruited for the study ( 6 males, mean age $22.250 \pm 2.543$ years, mean education $15.188 \pm 1.759$ years). All were native speakers of one of the Croatian dialects, students at the University of Zagreb, young adults, and right-handed as assessed by the Edinburgh Handedness Inventory (Oldfield 1971). Subjects who reported history of psychiatric or neurological disorders, uncorrected vision impairment, or more than one native language were not included in the study. Ethical approval was obtained by the Ethical Committee of the Faculty of Humanities and Social Sciences, University of Zagreb (dated 2018/10/03). All subjects signed an informed consent form.

\subsection{Verbal fluency assessment}

Subjects were to name as many words according to the given cue in 60 seconds. The categories animals and trees were used for SF, while the letters $\langle\mathrm{k}\rangle$ and $\langle\mathrm{m}\rangle$ (corresponding to phonemes $/ \mathrm{k} /$ and $/ \mathrm{m} /$ in Croatian dialects) were used for PF. An exploration of the Croatian web corpus hrWaC (Ljubešić \& Erjavec 2015) revealed that there were more lemmas starting with $\langle\mathrm{m}\rangle(\mathrm{N}=436719)$ compared to $\langle\mathrm{k}\rangle\left(\mathrm{N}=412\right.$ 961) (Goodness of fit: $\left.\chi^{2}{ }_{(1)}=664.300, \mathrm{p}<.00001\right)$. PF was administered one to two weeks after SF to avoid SF-related recency effects on PF. Responses were audiotaped and transcribed using ELAN (Version 5.8) (Wittenburg et al. 2006). Clusters were identified according to Troyer et al. (1997), with the cluster threshold set to 2 words. Two native speakers independently rated the outputs. Discrepancies were discussed until mutual agreement was achieved. Clustering and switching variables were calculated according to Gabrić \& Vandek (2020, in review). Dependent variables included: 1) legal words (raw), 2) perseveration rate (\%), 3) intrusion rate $(\%), 4)$ first-response latency $(\mathrm{ms}), 5)$ final silence (ms), 6) cluster magnitude 
(words per cluster), 7) switching rate $(\%), 8)$ between-cluster response latencies (ms), and 9) within-cluster response latencies (ms).

\subsection{Neuropsychological assessment}

To assess executive functioning, the Wisconsin Card Sorting Test (WCST) was administered using the PEBL battery of neuropsychological tests (Psychology Experiment Building Language, Version 2.0) (Mueller \& Piper 2014; Piper et al. 2012) on a computer. 128 trials were analyzed. Dependent variables included: 1) number of trials to complete the first subcategory (raw), 2) number of failures to maintain set (raw), and 3) learning to learn score. Failure to maintain set refers to the production of five successive correct responses but failing to make the 10 correct responses that are required to complete the category. Learning to learn was calculated as the average difference in percent errors between successive categories.

\subsection{Statistics}

Statistical analyses were performed in JASP (Version 0.11.1.0). Comparisons between the animal and tree variables, and $\mathrm{K}$ and $\mathrm{M}$ variables were performed using the paired-sample Wilcoxon signed-rank test. The intrusion rates on SF were compared with the one-sample Wilcoxon signedrank test with the test value set at $1 \pm 1$, as no subjects produced intrusions on the animal task. The

effect sizes are given by the matched rank biserial correlation. Correlations were explored using Spearman correlation coefficients.

\section{RESULTS}

A selection of the results is displayed in Figure 1. 


\subsection{Comparisons and correlations within semantic fluency tasks}

Results from the comparisons are displayed in Table 1. On the animal compared to the tree task, subjects produced significantly more legal words, had a significantly lower intrusion rate, significantly shorter first-response latencies and final silence periods, as well as significantly shorter between-cluster response latencies. All of the effect sizes but the one for final silence were strong. Additionally, subjects displayed significantly shorter within-cluster compared betweencluster response latencies on both the animal and tree tasks $\left(\mathrm{Z}=119.0, \mathrm{p}<.001, \mathrm{r}_{\mathrm{b}}=0.983\right.$ and $\mathrm{Z}$ $=120.0, \mathrm{p}<.001, \mathrm{r}_{\mathrm{b}}>.999$, respectively).

The number of legal words on both the animal and tree tasks was significantly positively correlated with cluster magnitude $(\rho=.547, \mathrm{p}=.035$ and $\rho=.753, \mathrm{p}=.001$, respectively).

Table 1. Within-sample comparisons between the animal and tree tasks

\begin{tabular}{lccc}
\hline & Animals & Trees & Statistics \\
\hline Legal words (raw) & 23.667 & 9.400 & $\mathrm{Z}=120.0, \mathrm{p}<.001, \mathrm{r}_{\mathrm{b}}>.999$ \\
& $(6.114)$ & $(2.293)$ & \\
Perseveration rate (\%) & 1.0 & 0.6 & $\mathrm{Z}=3.0, \mathrm{p}=.584, \mathrm{r}_{\mathrm{b}}=.400$ \\
& $(2.6)$ & $(2.2)$ & \\
Intrusion rate (\%)* & 0.0 & 4.7 & $\mathrm{Z}=21.0, \mathrm{p}=.036, \mathrm{r}_{\mathrm{b}}>.999$ \\
& $(0.0)$ & $(7.1)$ & \\
First-response latency (ms) & 1029.333 & 1789.333 & $\mathrm{Z}=11.0, \mathrm{p}=.006, \mathrm{r}_{\mathrm{b}}=.816$ \\
& $(360.723)$ & $(1064.341)$ & \\
Final silence (ms) & 5015.067 & 14217.067 & $\mathrm{Z}=22.0, \mathrm{p}=.030, \mathrm{r}_{\mathrm{b}}=.633$ \\
& $(3883.662)$ & $(12080.566)$ & \\
Cluster magnitude (words) & 3.331 & 2.961 & $\mathrm{Z}=81.0, \mathrm{p}=.244, \mathrm{r}_{\mathrm{b}}=.350$
\end{tabular}




\begin{tabular}{lccc} 
Switching rate $(\%)$ & 31.3 & 43.5 & $\mathrm{Z}=22.0, \mathrm{p}=.108, \mathrm{r}_{\mathrm{b}}=.516$ \\
& $(15.0)$ & $(14.6)$ & \\
Between-cluster response & 1706.500 & 5979.967 & $\mathrm{Z}=119.0, \mathrm{p}<.001, \mathrm{r}_{\mathrm{b}}=.983$ \\
latencies $(\mathrm{ms})$ & $(686.115)$ & $(3234.909)$ & \\
& 427.333 & 657.833 & $\mathrm{Z}=79.0, \mathrm{p}=.293, \mathrm{r}_{\mathrm{b}}=.317$ \\
$\begin{array}{l}\text { Within-cluster response } \\
\text { latencies }(\mathrm{ms})\end{array}$ & $(278.240)$ & $(563.643)$ & \\
\hline
\end{tabular}

Note: Mean values are reported. Standard deviations appear in parentheses. *one-sample Wilcoxon-signed rank test

\subsection{Comparisons and correlations within phonemic fluency tasks}

Results from the comparisons are displayed in Table 2. On the K compared to the M task, subjects produced significantly more legal words and had significantly shorter between-cluster response latencies. Additionally, there were no significant differences between the between-cluster and within-cluster response latencies on either the $\mathrm{K}$ or $\mathrm{M}$ task $\left(\mathrm{Z}=31.0, \mathrm{p}=.569, \mathrm{r}_{\mathrm{b}}=.205\right.$ and $\mathrm{Z}=$ $25.0, \mathrm{p}=.301, \mathrm{r}_{\mathrm{b}}=.359$, respectively).

Table 2. Within-sample comparisons between the $\mathrm{K}$ and $\mathrm{M}$ tasks

\begin{tabular}{lccc}
\hline & $\mathrm{K}$ & $\mathrm{M}$ & Statistics \\
\hline Legal words (raw) & 15.750 & 12.833 & $\mathrm{Z}=58.0, \mathrm{p}=.029, \mathrm{r}_{\mathrm{b}}=.758$ \\
& $(2.864)$ & $(4.086)$ & \\
Perseveration rate (\%) & 0.4 & 1.2 & $\mathrm{Z}=0.0, \mathrm{p}=.371, \mathrm{r}_{\mathrm{b}}>.999$ \\
& $(1.3)$ & $(2.9)$ & $\mathrm{N} / \mathrm{A}$ \\
Intrusion rate (\%) & 0.0 & 0.0 & \\
& $(0.0)$ & $(0.0)$ & $\mathrm{Z}=50.0, \mathrm{p}=.424, \mathrm{r}_{\mathrm{b}}=.282$ \\
First-response latency (ms) & 2409.167 & 1893.333 & \\
& $(1709.005)$ & $(640.289)$ & \\
Final silence (ms) & 3740.500 & 6285.333 & $\mathrm{Z}=30.0, \mathrm{p}=.519, \mathrm{r}_{\mathrm{b}}=.231$ \\
& $(3270.119)$ & $(6335.809)$ & \\
Cluster magnitude (words) & 2.562 & 2.540 & $\mathrm{Z}=25.0, \mathrm{p}=.813, \mathrm{r}_{\mathrm{b}}=.111$
\end{tabular}




\begin{tabular}{lccc} 
Switching rate $(\%)$ & 72.3 & 71.4 & $\mathrm{Z}=29.000, \mathrm{p}=.756, \mathrm{r}_{\mathrm{b}}=.121$ \\
& $(13.9)$ & $(11.4)$ & \\
Between-cluster response & 2014.583 & 2732.708 & $\mathrm{Z}=9.0, \mathrm{p}=.016, \mathrm{r}_{\mathrm{b}}=.769$ \\
latencies (ms) & $(787.570)$ & $(1158.775)$ & \\
& & & \\
Within-cluster response & 1864.375 & 2169.375 & $\mathrm{Z}=39.0, \mathrm{p}>.999, \mathrm{r}_{\mathrm{b}}<.001$ \\
latencies (ms) & $(1347.603)$ & $(1698.251)$ & \\
\hline
\end{tabular}

Note: Mean values are reported. Standard deviations appear in parentheses.

The number of legal words on the K task was significantly positively and strongly correlated with cluster magnitude $(\rho=.731, \mathrm{p}=.007)$, while on the $\mathrm{M}$ task none of the correlations were significant.

\subsection{Correlations with sociodemographic variables}

As expected in a sample of university students, age and education were significantly positively and strongly correlated $(\rho=.746, p<.001)$.

Both age and education were significantly positively and moderately correlated with cluster magnitude on the animal task $(\rho=.659, \mathrm{p}=.008$ and $\rho=.689, \mathrm{p}=.005$, respectively) but there were no significant correlations between the tree task and sociodemographic variables.

Age was significantly negatively and moderately correlated with the first-response latency on the $\mathrm{K}$ task $(\rho=-.623, \mathrm{p}=.030)$. There were no significant correlations between the $\mathrm{M}$ task and sociodemographic variables.

\subsection{Correlations with neuropsychological variables}

On the animal task, the first-response latency and switching rate were significantly correlated with the number of trials to complete the first subcategory on WCST $(\rho=.682, p=.005$ and $\rho=-.523$, 
$\mathrm{p}=.045$, respectively). On the tree task, productivity was significantly moderately correlated with the learning to learn score $(\rho=.574, p=.025)$ and the number of trials to complete the first subcategory $(\rho=-.527, p=.044)$. Furthermore, cluster magnitude was significantly positively and strongly correlated with the learning to learn score $(\rho=.799, p<.001)$.

Within-cluster response times on the $\mathrm{M}$ task were significantly positively and moderately correlated with the number of trials to complete the first subcategory $(\rho=.627, p=.029)$. There were no significant correlations between the K task and WCST variables.

a) CORRECT WORDS

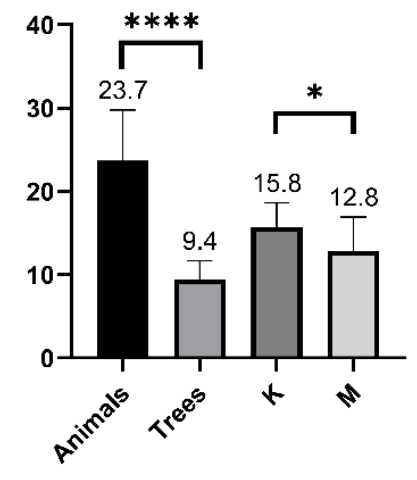

c) FIRST RESPONSE LATENCY

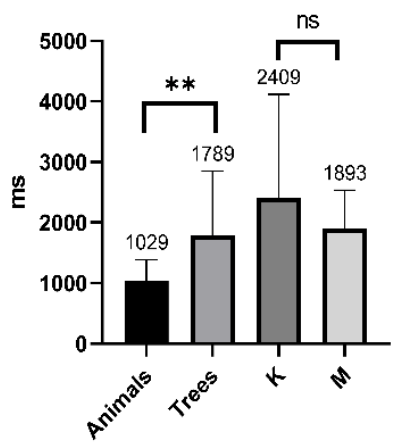

b) INTRUSION RATE

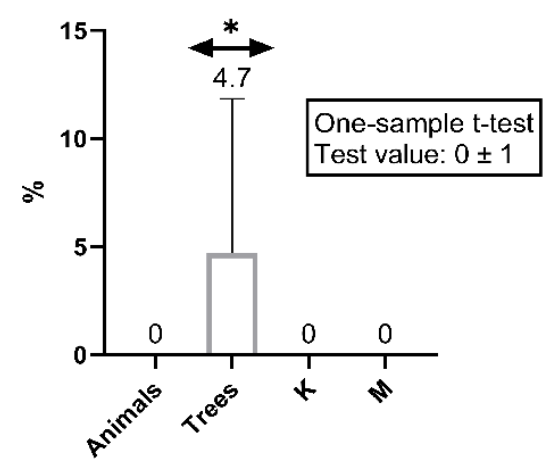

d)

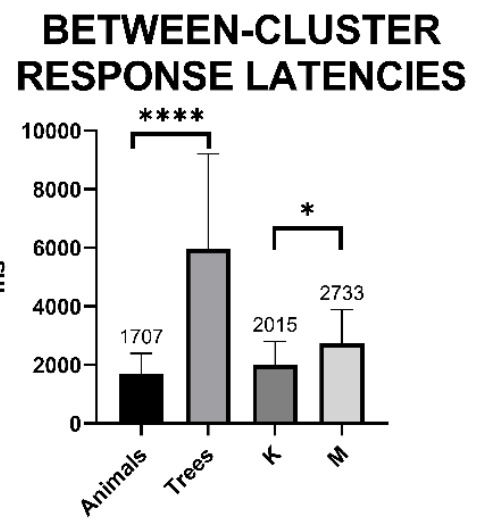

Figure 1. Selection of the results from the comparisons between SF and between PF tasks.

\section{DISCUSSION}




\subsection{Semantic fluency}

Comparisons between the animal and tree fluency tasks revealed highly different performance patterns across the two tasks. Subjects produced significantly $60.28 \%$ more legal words on the animal compared to the tree category, suggesting that the subjects in our sample had had access to more animal than tree words, as well as that the animal task was appreciably less difficult compared to the tree task. Both the former and the latter suggestions are also arguably indicated by the significant differences in the duration of the final silence between the two tasks. On average, subjects stopped responding in the 55. second on the animal task compared to the 46. second on the tree task, with the latter possibly indicating that the readily available tree words were more or less exhausted before the end of the task and that the access to less familiar (but entrenched) words was much more demanding in the tree compared to the animal task. Subjects were further significantly slower to utter the first response on the tree compared to the animal task, indicating that it was more difficult for the subjects to access the category trees, retrieve, and utter the first tree word compared to the category animals. The results regarding first-response latency and final silence indicate that the tree task was more difficult for the subjects compared to the animal task during both the incipient and ending stages of the task.

Significant differences between the two tasks were identified in the intrusion rate as well. While no subjects produced intrusions on the animal task, intrusions on the tree task were produced by seven subjects. We would like to note at this point that we were very careful in classifying responses as intrusions and that our criteria for intrusion classification were more lax (but nevertheless precise) compared to, for example, Moreno-Martínez et al. (2017) who classified fruit trees (such as pear) as intrusions on the tree task. In our study, fruit trees were classified as legal because we found there was no reason to classify them as errors. Intrusions on the tree task 
included: glog 'hawthorn' (2), bugenvilija 'bougainvillea' (1), hortenzija 'hortensia' (1), ebanovina 'ebony' (1), ruža 'rose' (1), jorgovan 'lilac' (1), loza 'grapevine', and žir 'acorn' (1). The significantly higher intrusion rate on the tree compared to the animal task indicates that the boundaries of the category trees are considerably looser compared to the boundaries of the category animals in our sample.

Subjects did not exhibit significant differences in the clustering and switching strategies between the two SF tasks. However, while descriptive data showed only slight differences in cluster magnitude, the differences in the switching rate were more pronounced, suggesting that subjects were less likely to produce a cluster once a switch has been uttered on the tree compared to the animal task. This might reflect lesser functional connectivity between concepts in the category trees compared to animals and, specifically, lesser hypernymic/hyponymic organization within the category trees compared to animals. In any case, the existence of clusters on the tree task still indicates the presence of hypernymic/hyponymic and co-hyponymic structures in the category trees. Subjects were significantly slower in between-cluster response latencies on the tree compared to the animal task indicating that the functional connectivity between subcategories is less efficient in the category trees compared to animals. Interestingly, larger clusters were associated with better productivity on both the animal and tree tasks, yet the correlation coefficient was considerably higher in the tree task. Thus, those who had better organization of hypernymic/hyponymic and co-hyponymic relations between concepts in both categories produced more words on the given SF task.

Correlational analyses further revealed disproportionate associations between different WCST variables and the two SF tasks. While subjects who made fewer errors in the first subcategory on the WCST switched at higher rates on animal fluency, the learning to learn score was significantly 
positively and strongly correlated with cluster magnitude on the tree task, indicating subjects who displayed improvement in the error rates across the task's subcategories built larger tree clusters. The former result may not be surprising given that it has already been proposed that switching on $\mathrm{SF}$ is a more controlled process, yet the latter is contrary to suggestions that clustering is a rather automatic process (Troyer \& Moscovitch 2006). Our results suggest that the automaticity of clustering may depend on the given semantic category and that clustering may underlie more controlled processes when more demanding semantic categories are administered.

\subsection{Phonemic fluency}

The differences in performance patterns between the two PF tasks were much less pronounced than between the two SF tasks. Significant differences were observed in the productivity and between-cluster response latencies, with subjects producing significantly more words and having significantly shorter between-cluster response latencies on the K compared to the M task. These results suggest that performance on the K task was easier for the subjects compared to the M task. Because there are presumably more words starting with $\langle\mathrm{m}\rangle$ compared to the $\langle\mathrm{k}\rangle$ task, this result presumably does not reflect the disproportionate number of words starting with either $\langle\mathrm{k}\rangle$ or $\langle\mathrm{m}\rangle$ in the Croatian dialects.

Somewhat unexpectedly, the only significant correlation between PF and WCST variables was between within-cluster response times on the $\mathrm{M}$ task and the number of trials to complete the first subcategory. These results suggest that the executive demands were similar between the two PF tasks, yet considerably lower compared to the two SF tasks. 


\section{CONCLUSIONS}

Our results show that there are stark differences in performance patterns and associations with the WCST within different SF and PF tasks. Our results have important implications for research using VF tasks as they indicate that researchers should carefully select the semantic categories and phonemes/letters that are to be administered within their neuropsychological assessments.

\section{References}

Aita, S.L., Beach, J.D., Taylor, S.E., Borgogna, N.C., Harrell, M.N., \& Hill, B.D. (2018). Executive, language, or both? An examination of the construct validity of verbal fluency measures. Applied Neuropsychology: Adult, $\quad$ 441-451. https://doi.org/10.1080/23279095.2018.1439830

Amunts, J., Camilleri, J.A., Eickhoff, S.B., Heim, S., \& Weis, S. (2020). Executive functions predict verbal fluency scores in healthy participants. Scientific Reports, 10, 11141. https://doi.org/10.1038/s41598-020-65525-9

Barry, D., Bates, M.E., \& Labouvie, E. (2008). FAS and CFL forms of verbal fluency differ in difficulty: A meta-analytic study. Applied Neuropsychology, 15(2), 97-106. https://doi.org/10.1080/09084280802083863

Borkowski, J.G., Benton, A.L., \& Spreen, O. (1967). Word fluency and brain damage. Neuropsychologia, 5(2), 135-140. https://doi.org/10.1016/0028-3932(67)90015-2 
Caramazza, A., \& Shelton, J. R. (1998). Domain-specific knowledge systems in the brain: The animate-inanimate distinction. Journal of Cognitive Neuroscience, 10(1), 1-34. https://doi.org/10.1162/089892998563752

Diamond, A. (2013). Executive functions. Annual Review of Psychology, 64(1), 135-168. https://doi.org/10.1146/annurev-psych-113011-143750

Diaz, M., Sailor, K., Cheung, D., \& Kuslansky, G. (2004). Category size effects in semantic and letter fluency in Alzheimer's patients. Brain and Language, 89, 108-14. https://doi.org/10.1016/S0093-934X(03)00307-9

Gabrić, P., \& Vandek, M. (2020). Semantic fluency reveals reduced functional connectivity between subcategorical co-hyponyms in recent-onset inpatients with first-episode psychosis [Preprint]. PsyArXiv. https://doi.org/10.31234/osf.io/75htx

Gabrić, P., \& Vandek, M. (in review). Semantic fluency reveals reduced functional connectivity between subcategorical co-hyponyms in recent-onset inpatients with first-episode psychosis. Clinical Linguistics \& Phonetics.

Henry, J., \& Crawford, J. (2005). A meta-analytic review of verbal fluency deficits in schizophrenia relative to other neurocognitive deficits. Cognitive Neuropsychiatry, 10(1), 1-33. https://doi.org/10.1080/13546800344000309

Henry, L.A., Messer, D.J., \& Nash, G. (2015). Executive functioning and verbal fluency in children with language difficulties. Learning and Instruction, 39, 137-147. https://doi.org/10.1016/j.learninstruc.2015.06.001 
Huth, A.G., de Heer, W.A., Griffiths, T.L., Theunissen, F.E., \& Gallant, J.L. (2016). Natural speech reveals the semantic maps that tile human cerebral cortex. Nature, 532, 453-458. https://doi.org/10.1038/nature17637

Jebahi, F., Jaoude, R.A., \& Ellis, C. (2020). Semantic verbal fluency task: The effects of age, educational level, and sex in Lebanese-speaking adults. Applied Neuropsychology: Adult (Latest Articles). https://doi.org/10.1080/23279095.2020.1821031

Lago, S., Scharinger, M., Kronrod, Y., \& Idsardi, W.J. (2015). Categorical effects in fricative perception are reflected in cortical source information. Brain and Language, 143, 52-58. https://doi.org/10.1016/j.bandl.2015.02.003

Luo, L., Luk, G., \& Bialystok, E. (2010). Effect of language proficiency and executive control on verbal fluency performance in bilinguals. Cognition, 114(1), 29-41. https://doi.org/10.1016/j.cognition.2009.08.014

Ljubešić, N., \& Erjavec, T. (2011). hrWaC and slWaC: compiling web corpora for Croatian and Slovene. In Habernal, I., \& Matoušek, V. (Eds.), Text, Speech and Dialogue. TSD 2011. Lecture Notes in Computer Science, vol 6836. Springer, 395-402. https://doi.org/10.1007/978-3-64223538-2_50

Moreno-Martínez, F.J., Ruiz, M., \& Montoro, P.R. (2017). Why almost always animals? Ranking fluency tasks for the detection of dementia based on receiver operating characteristic (ROC) and quality ROC analyses. Dementia and Geriatric Cognitive Disorders, 43, 59-70. https://doi.org/10.1159/000454916 
Mueller, S.T., \& Piper, B.J. (2014). The Psychology Experiment Building Language (PEBL) and PEBL Test Battery. Journal of Neuroscience Methods, 222, 250-259. https://doi.org/10.1016/j.jneumeth.2013.10.024

Neves, T.R.F., de Araújo, N.B., de Oliveira Silva, F., Ferreira, J.V.A., Nielsen, T.R., Engedal, K., ..., \& Deslandes, A.C. (2020). Accuracy of the semantic fluency test to separate healthy old people from patients with Alzheimer's disease in a low education population. Jornal Brasileiro de Psiquiatria, 69(2), 82-87. https://doi.org/10.1590/0047-2085000000270

Oldfield, R.C. (1971). The assessment and analysis of handedness: The Edinburgh inventory. Neuropsychologia, 9, 97-113. https://doi.org/10.1016/0028-3932(71)90067-4

Piper, B.J., Li, V., Eiwaz, M.A., Kobel, Y.V., Benice, T.S., Chu, A.M., .., \& Mueller, S.T. (2012) Executive function on the Psychology Experiment Building Language tests. Behavior Research Methods, 44, 110-123. https://doi.org/10.3758/s13428-011-0096-6

Raucher-Chéné, D., Achim, A.M., Kaladjian, A., \& Besche-Richard, C. (2017). Verbal fluency in bipolar disorders: A systematic review and meta-analysis. Journal of Affective Disorders, 207, 359-366. https://doi.org/10.1016/j.jad.2016.09.039

Sekulić Sović, M., Erdeljac, V., Kužina, I., Vandek, M., Mimica, N., Ostojić, D., \& Savić, A. (2019) Shared lexical-semantic features and the animacy effect in early-course psychosis. In Erdeljac, V., \& Sekulić Sović, M. (Eds.), Interdisciplinary Linguistic and Psychiatric Research on Language Disorders (pp. 69-81). FF Press. https://doi.org/10.17234/9789531758314.06 
Shao, Z., Janse, E., Visser, K., \& Meyer, A.S. (2014). What do verbal fluency tasks measure? Predictors of verbal fluency performance in older adults. Frontiers in Psychology, 5, 772. https://doi.org/10.3389/fpsyg.2014.00772

Tagini, S., Seyed-Allaei, S., Scarpina, F., Toraldo, A., Mauro, A., Cherubini, P., \& Reverberi, C. (2018). When fruits lose to animals: Disorganized search of semantic memory in Parkinson's disease. Neuropsychology (Advance online publication). https://doi.org/10.1037/neu0000429

Tan, E.J., Neill, E., Tomlinson, K., \& Rossell, S.L. (2020). Semantic memory impairment across the schizophrenia continuum: A meta-analysis of category fluency performance. Schizophrenia Bulletin Open, 1(1), sgaa054. https://doi.org/10.1093/schizbullopen/sgaa054

Thiele, K., Quinting, J.M., \& Stenneken, P. (2016). New ways to analyze word generation performance in brain injury: A systematic review and meta-analysis of additional performance measures. Journal of Clinical and Experimental Neuropsychology, 38(7), 764-781. https://doi.org/10.1080/13803395.2016.1163327

Troyer, A.K., Moscovitch, M., \& Winocur, G. (1997). Clustering and switching as two components of verbal fluency: Evidence from younger and older healthy adults. Neuropsychology, 11(1), 138-146. https://doi.org/10.1037//0894-4105.11.1.138

Troyer, A.K., \& Moscovitch, M. (2006). Cognitive processes of verbal fluency tasks. In A.M. Poreh (Ed.), The Quantified Process Approach to Neuropsychological Assessment (pp. 143160). Taylor \& Francis. https://psycnet.apa.org/record/2007-16443-009 
Vandek, M., Gabrić, P., Kužina, I., Erdeljac, V., \& Sekulić Sović, M. (2018, July 2-4). Verbal fluency and working memory interaction [Conference poster]. 10th International Workshop on Language Production, Nijmegen, Netherlands. https://doi.org/10.17605/osf.io/au6vd

Ventura, P., Morais, J., \& Kolinsky, R. (2005). Evaluating feature-category relations using semantic fluency tasks. Brain and Cognition, 58, 202-212. https://doi.org/10.1016/j.bandc.2004.11.004

Whiteside, D.M., Kealey, T., Semla, M., Luu, H., Rice, L., Basso, M.R., \& Roper, B. (2016). Verbal fluency: Language or executive function measure?. Applied Neuropsychology: Adult, 23(1), 29-34. https://doi.org/10.1080/23279095.2015.1004574

Wittenburg, P., Brugman, H., Russel, A., Klassmann, A., \& Sloetjes, H. (2006). ELAN: A professional framework for multimodality research. In Calzolari, N. et al. (Eds.), Proceedings of the Fifth International Conference on Language Resources and Evaluation (LREC'06) (pp. 15561559). European Language Resources Association (ELRA). http://www.lrecconf.org/proceedings//rec2006/pdf/153_pdf.pdf 